\title{
SESSYL : THE STUDY OF THE POSITIONING SYSTEMS FOR ROAD CONSTRUCTION ROBOTICS
}

François Peyret

Site Robotics Section, Laboratoire Central des Ponts et Chaussées, BP 19, 44340, Bouguenais, FRANCE

\begin{abstract}
The first part of the paper gives a definition of positioning in the context of road work sites and stresses the advantages brought by the new positioning systems. A logical classification of these systems is also proposed. One technique in particular which seems to be most promising at the moment : the Global Positioning System (GPS), is detailed. Then the new test facility for positioning systems, existing at the "Laboratoire Central des Ponts et Chaussées" (Central Laboratory of Bridges and Roads) is described, our Laboratory having decided to play a major role in the evaluation and development of systems adapted to road construction. At last, some results from recent tests performed on a Ultra Precise Differential GPS on a kinematic mode are given. These tests have already produced some interesting results that would have been hard to obtain without the test facility.
\end{abstract}

\section{WHAT IS POSITIONING ON A ROAD CONSTRUCTION SITE?}

The term "positioning" (or "localization") such as it is used in this article signifies :

measurement in real-time, of one or several position or/and attitude parameters of a construction piece of equipment or tool.

For example: the plan position of a compactor, the data set (position + height + cros. slope) of a finisher screed, etc.

The notion of "real time" signifies that this information must be available in the form of a signal, at the moment and at the place where the work is being carried out, at a rate of at least a couple of Hertz, compatible with the evolution speed of the current machines.

Actually, a positioning system offers a very precious thing : a link between the work site phaze and the design phaze.

Compared to the traditional way, a new methodology of managing and carrying out the work can be imagined because of this link .

Figure 1 shows what the future methodology could be, using positioning systems iniegrated with adapted software, and fully using the "project" numeric data received from the CAD design [1]. 
It can be seen that the CAD software becomes more than a simple design software, but also an interactive geometric database in which the two models (theoretical model of the project and the actual model of the work as-built) co-exist.

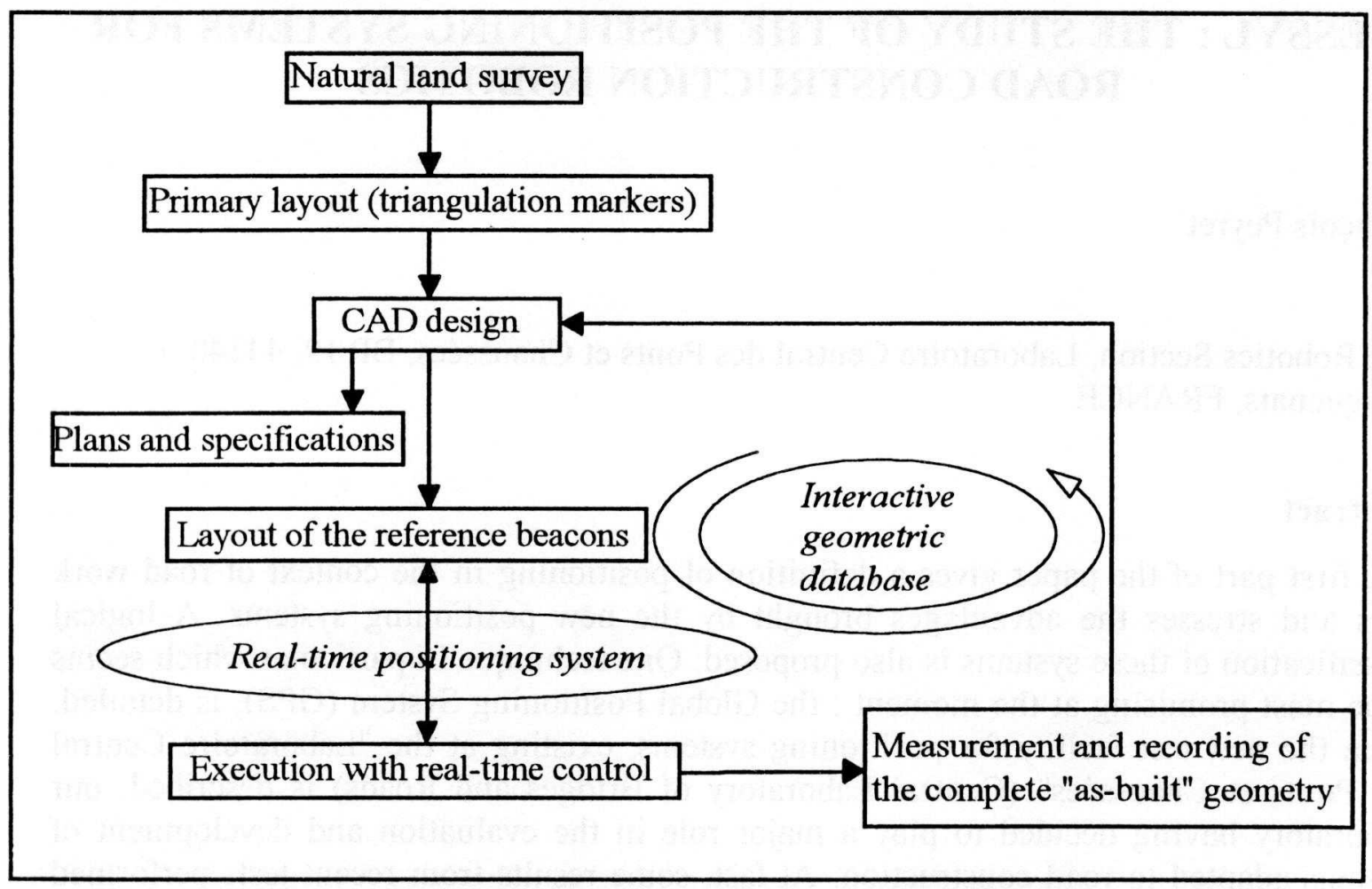

Fig. 1 : Future methodology using real-time positioning

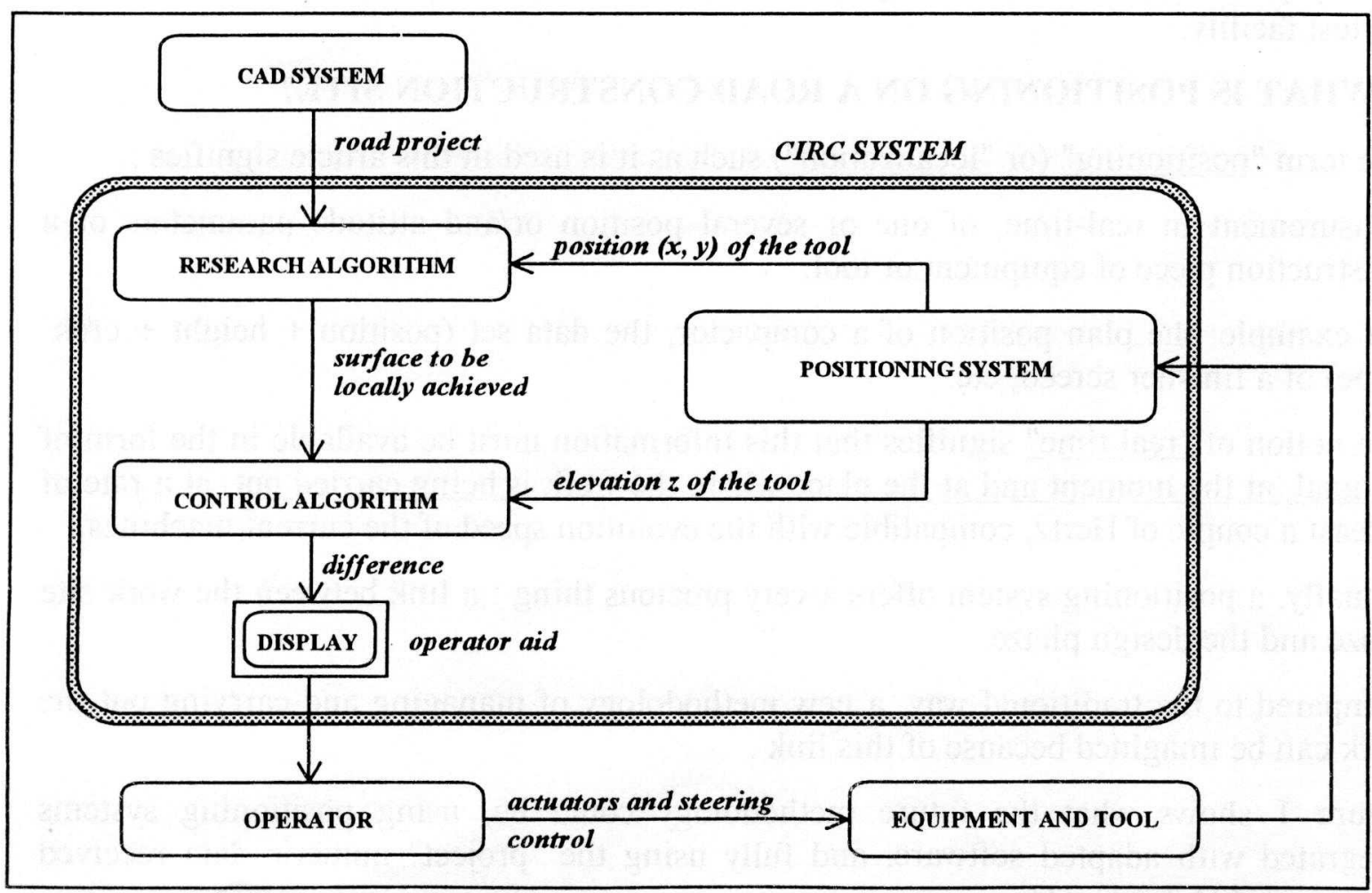

Fig. 2 : Diagram of a Computer Integrated Road Construction System (CIRC) 
The plans are only used as work and dialogue aids and no longer as links in the chain of data transmission.

It is also to be noted that the design-execution-control phases become closely linked together (in a loop) and share the same unique data located in the database, reducing the risks of transmission errors to nearly zero.

In conclusion, it appears evident that this technological addition of real-time positioning will open the door to a new concept of methodology which could be termed "Computer Integrated Road Construction" (CIRC) [2].

An example of a "Computer Integrated Road Construction" (CIRC) system applied to profiling/levelling equipment using an operator aiding type interface is schematically shown in figure 2 .

\section{GENERAL CLASSIFICATION OF POSITIONING METHODS}

\subsection{Positioning by dead reckoning and absolute positioning}

Two main groups of methods can be identified [3]:

$\sigma$ Positioning methods referred to as "dead reckoning" where the current position is determined by integration of successively oriented movements from the initial position, using data provided by sensors which are generally proprioceptive (odometers, gyrometers, etc.).

Positioning methods referred to as "absolute" where the current position is determined by measurements carried out on markers (or beacons) with known positions. These beacons are generally situated close to the piece of equipment (a few meters to a few kilometers) except in the exceptional case where they are artificial satellites orbiting the Earth (GPS).

These two groups each possess characteristic advantages and disadvantages which make them appear more complementary than concurrent.

In the remainder of this article, we shall only describe the absolute positioning systems which we shall call "beacon systems". These seem to us to be of greater interest than those in the first group for one main reasons :

- the drift of precision of the dead-reckoning systems very often forces them to be corrected with the help of beacon systems - this makes these latest essential on work sites.

\subsection{Classification of the methods of positioning by beacons}

\subsubsection{General principle}

$\square$ Several variants exist and several technologies can be used.

The discriminant factors are :

the nature of the beacons, the type and number of geometrical magnitudes measured, the nature of the wave serving as measuring vector. 


\subsubsection{Nature of the beacons}

These can be either active (transmitting the wave), semi-active (equipped with an active sensor), or passive (only reflecting the wave).

\subsubsection{Type of parameters measured}

Two types of geometrical data can be measured :

distances - this means range-finding for the basic measurements and trilateration for calculating position.

angles - this means goniometry for the measurements and triangulation for calculation.

Depending on the number of independent parameters measured, you can compute your position according to the following rule : $\rightarrow$ The measurement of $N$ independent parameters allows $\boldsymbol{N}$ degrees of freedom to be calculated .

\subsubsection{Nature of the wave used}

The waves used for measurement can be of 3 types:

Ultrasonic waves, limited to a short range of a few decameters.

Light waves, visible or invisible (infrared), either coherent light (laser) or noncoherent.

The range can reach several kilometers. This technology is already in current use on work sites in the form of surveying and static layout devices.

Microwaves, generally centimetric (few GHz).

The range can be quasi unlimited for satellite based systems but are of the order of several tens or hundreds of $\mathrm{km}$ for land beacon systems.

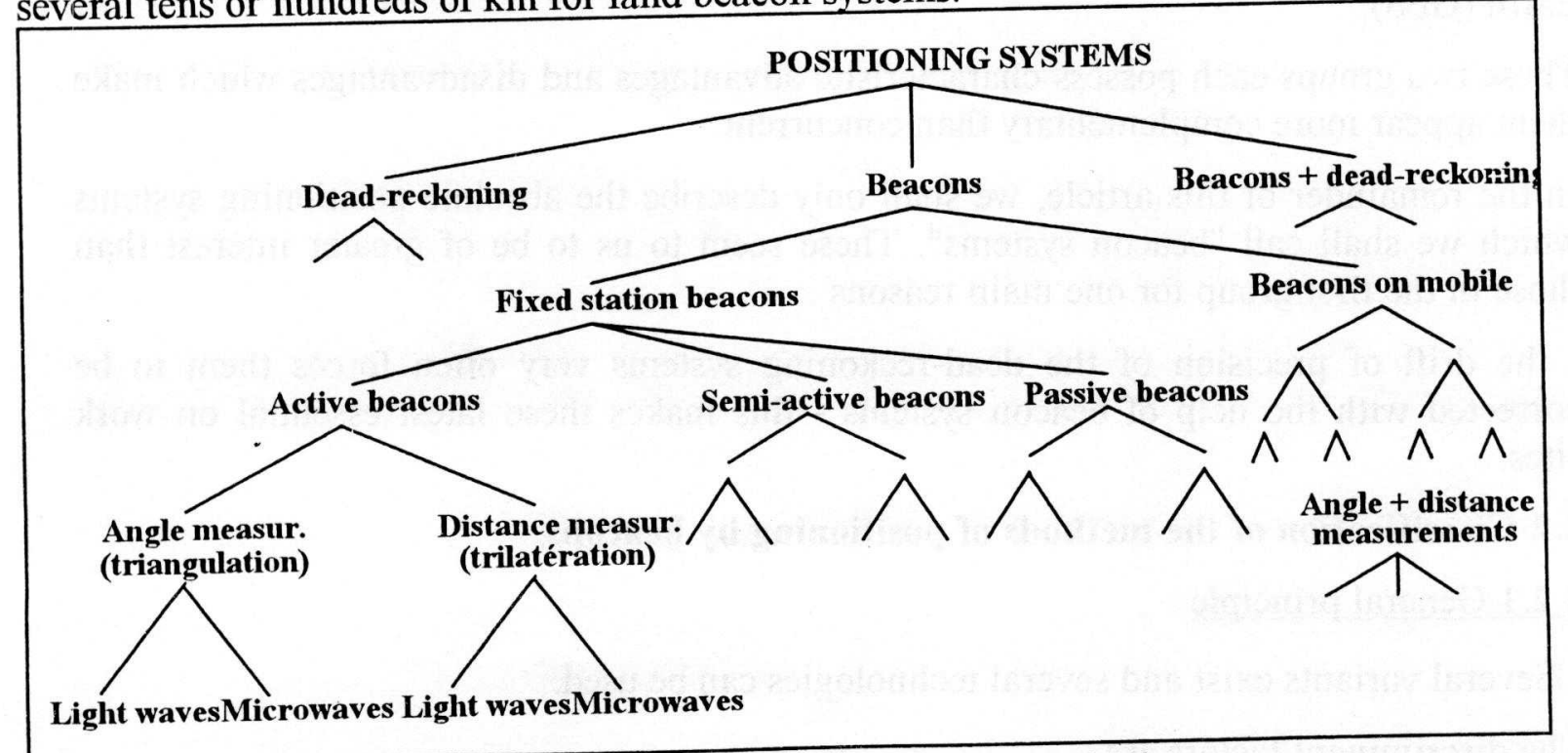

Fig. 3 : Tree diagram of positioning methods

The combination of different discriminant factors allows multiple configurations to be envisaged and which can generally be physically realized. Part of the branches of the graph of possible combinations is represented in figure 3. 


\section{GPS (Global Positioning System)}

\subsection{General points}

Of the new positioning techniques discussed today, it is clear that GPS is at the forefront. According to our classification, this consists of a system :

- using active beacons and whose position is assumed to be perfectly known at every moment - this allows it to be classified with the fixed station beacons systems.

- of trilateration type,

- using microwaves.

Many things have been said and written about its performances and this arouses great confusion in the uninformed reader, considering the countless configurations and modes of use which exist and do not cease to be developed [4].

Figure 4 summarizes the main modes, their accuracy and their special field of application.

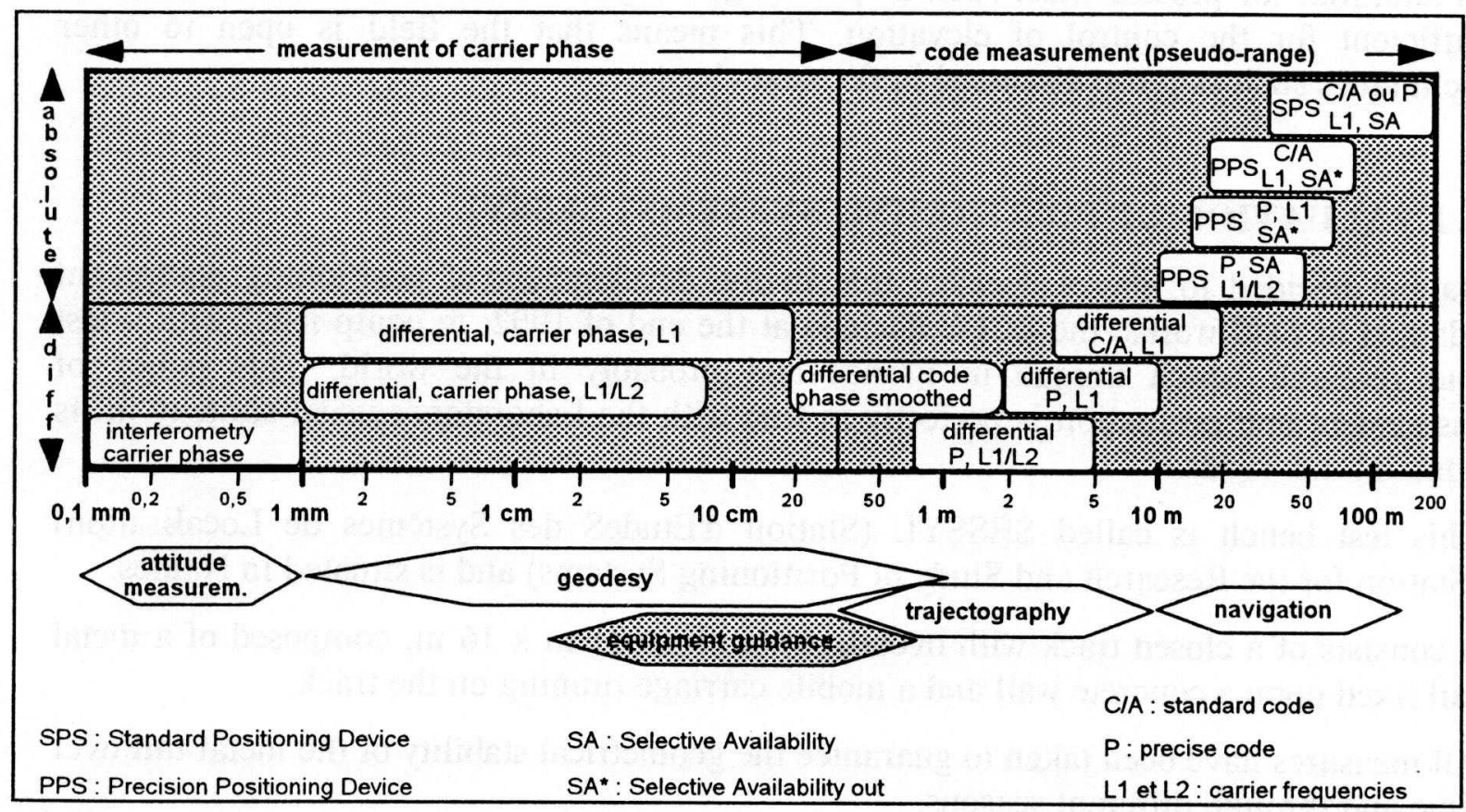

Fig. 4 : Modes of use and applications of GPS

\subsection{Use of GPS for positioning/guidance of work site equipment}

The modes which are of interest to us are those which can function in real-time and which provide centimetric or decimetric accuracy. These correspond to real-time use of the "differential carrier phase" modes situated in the lower left quadrant of the diagram.

These modes of use are today the subject of important research and development work for the main manufacturers in the field (ASHTECH, SERCEL, TRIMELE, etc.) and the first products appeared in 1994. 
To put this type of system into operation requires the following configuration :

- a fixed station reference receiver, communicating its phase measurements by means of Hertzian waves to the mobile receiver,

- a mobile receiver fitted to the point to be localized, linked to a microprocessor set to carry out real-time processing.

One of the first products available in France (K.A.R.T. by SERCEL) is given for an accuracy better than $5 \mathrm{~cm}$ (within two standard deviations), a calculation frequency of $1.7 \mathrm{~Hz}$ and a price of the order of $\$ 53000$ without computer.

The major technical problem to be resolved in this mode of use is the phase ambiguity resolution and the correction procedures in the case of momentary loss of signals (it is necessary to receive continuously at least four satellites).

Despite the capabilities achieved in terms of electronics and signal processing (Kalman filter with 21 state variables...), the technique is reaching its limits and the risk of momentary loss of data for a few minutes will probably remain (in addition to the cost) one of the major drawbacks of these systems.

In addition, for precise work such as profiling, the present centimetric accuracy is not sufficient for the control of elevation. This means that the field is open to other techniques such as those discussed in the next chapter.

\section{EVALUATION OF POSITIONING SYSTEMS : SESSYL}

Having decided to play a decisive role in the development of positioning equipment adapted to road works, the LCPC decided at the end of 1992, to equip itself with a test and research bench unique in France and probably in the world. This policy of assessment and evaluation is perfectly in line with the Laboratory's usual strategy in its domain, i.e. roads.

This test bench is called SESSYL (Station d'EtudeS des Systčmes de Localisation) (Station for the Research and Study of Positioning Systems) and is situated in Nantes.

It consists of a closed track with overall dimensions $81 \mathrm{~m} \mathrm{x} 16 \mathrm{~m}$, composed of a metal rail fixed upon a concrete wall and a mobile carriage running on the track.

All measures have been taken to guarantee the geometrical stability of the metal rail over time and through different seasons.

The carriage is shown in figure 5 .

This carriage comprises on its upper part a platform which is mobile in height, roll and pitch, thus allowing a path to be described in space, for any positioning system loaded, and producing the six degrees of freedom of the system with respect to a reference frame. This reference frame could be either local, or national (Lambert + NGF), or world-wide (e.g. WGS 84 for the testing of GPS receivers).

All the degrees of freedom are programmable and different test paths can be used, the actuators control and the acquisition of all measurements being entirely automatic. The degrees of freedom X, Y, and Heading are of course coupled by the track itself. 


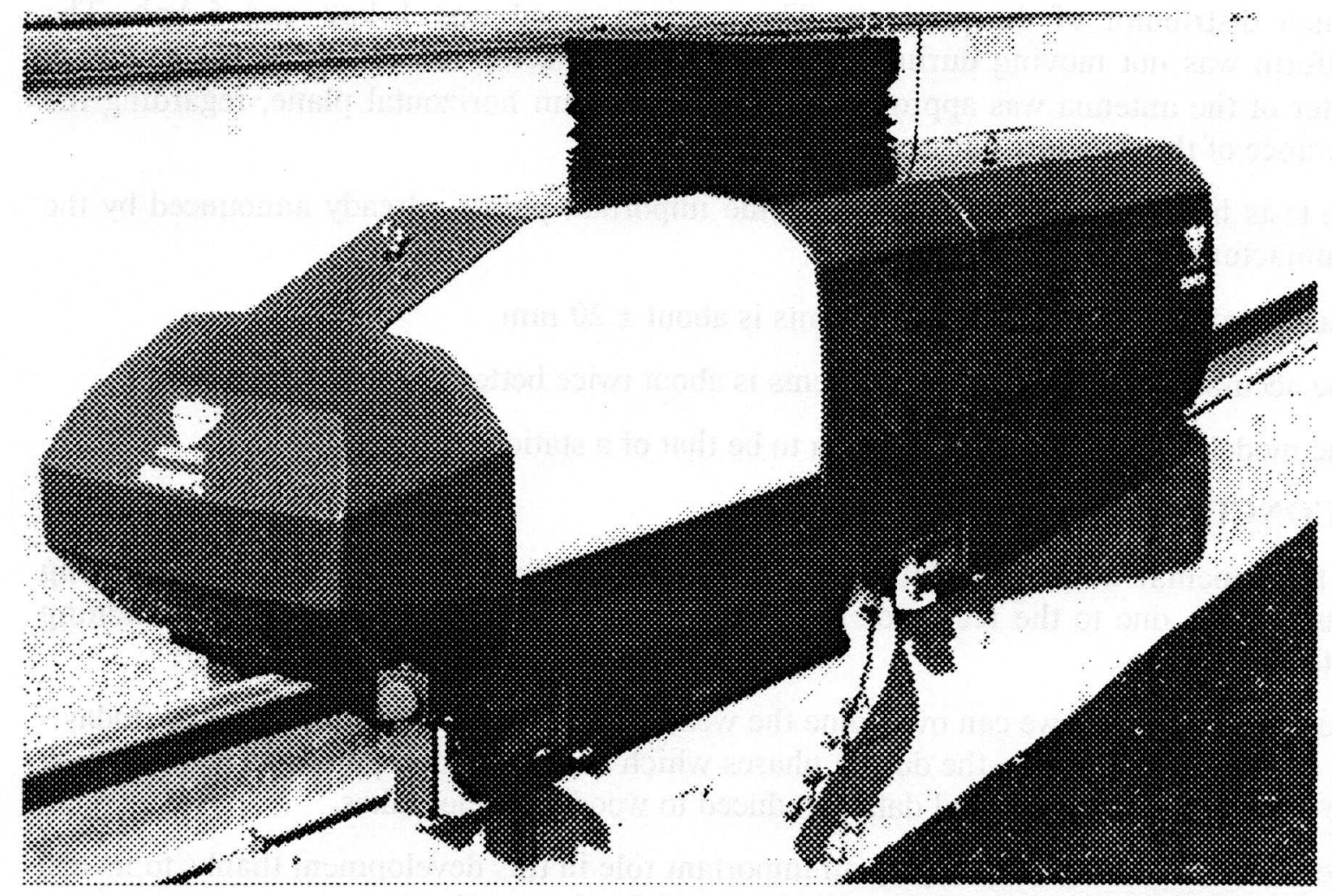

Fig 5 : The SESSYL carriage on its track

The movements and the available accuracies are indicated in figure 12.

\begin{tabular}{|l|l|l|l|l|l|l|}
\hline $\mathrm{D}^{\circ}$ of freedom & $\mathrm{X}$ & $\mathrm{Y}$ & $\mathrm{Z}$ & Heading & Roll & Pitch \\
\hline Movement & $81 \mathrm{~m}$ & $16 \mathrm{~m}$ & $300 \mathrm{~mm}$ & $360^{\circ}$ & $\pm 6^{\circ}$ & $\pm 6^{\circ}$ \\
\hline Accuracy & $< \pm 1 \mathrm{~cm}$ & $< \pm 1 \mathrm{~cm}$ & $< \pm 1 \mathrm{~mm}$ & $< \pm 0.1^{\circ}$ & $< \pm 0.01^{\circ}$ & $< \pm 0.01^{\circ}$ \\
\hline
\end{tabular}

Fig. 6 : The paths and accuracies of SESSYL

Two ranges of speed are available:

- a range corresponding to the speeds of levelling/profiling equipment : from 0 to 1 $\mathrm{km} / \mathrm{h}$,

- a range corresponding to the speeds of earth moving, surfacing and maintenance equipment : from 1 to $20 \mathrm{~km} / \mathrm{h}$.

The loadable mass can be $25 \mathrm{~kg}$ maximum and the carriage mass itself of the order of $450 \mathrm{~kg}$.

By early 1995 the carriage is still in the finalizing phase. It will be totally operational in a few months .

\section{EXAMPLE OF A TEST PERFORMED WITH SESSYL}

In November 1994 were performed a couple of dynamic experiments on our test facility SESSYL. The positioning system consisted in a set of two GPS receivers from Ashtech (Z 12), working in a carrier phase differential mode, with a post-processing of the kinematic data. The tests were carried out with the collaboration of Techmation, the 
French distributor of the products. The carriage speed was $1 \mathrm{kph}$ and $5 \mathrm{kph}$. The platform was not moving during the tests, so the trajectory described by the reception center of the antenna was approximately in an $\pm 2 \mathrm{~mm}$ horizontal plane, regarding the tolerance of the structure construction.

The tests have allowed us to confirm some important points, already announced by the manufacturer :

- the global accuracy in $\mathrm{Z}$ measurements is about $\pm 20 \mathrm{~mm}$,

- the accuracy in $\mathrm{X}$ and $\mathrm{Y}$ measurements is about twice better than the accuracy in $\mathrm{Z}$,

- the model of the error does not seem to be that of a stationary noise.

\section{CONCLUSION}

A fundamental turning point is taking place in the field of Civil Engineering construction, due to the recent or future arrival on the market of real-time positioning systems.

These systems mean we can overcome the weakness existing on construction sites today i.e. the huge gap between the design phases which are highly computerized and the work site itself where all numerical data is reduced to wooden grade stakes.

The French LCPC intends to play an important role in this development thanks to its test bed SESSYL which has been specially designed for this application.

\section{REFERENCES}

[1] Beliveau Y.J. et al. 3-D positioning for construction surveying and automation

Construction Congress II., ASCE, Boston, Mass, pp. 656-661, April 1991

[2] Peyret F. et Philippe H.

Towards Computer Integrated Construction

$9^{\text {th }}$ ISARC, Tokyo, pp. 859-868, June 1992

[3] Fargeon C. et Quin J.P.

Robotique Mobile

Ouvrage collectif, Teknea, Toulouse, pp. 75-85, 1993

[4] Peyret F.

Journée GPS du 11 Février 1992 au LCPC Nantes

Rapport interne LCPC, 13 p., Février 1992 